\title{
TITLE:
}

\section{Translational diffusion coefficient of wormlike regular three-arm stars}

$\operatorname{AUTHOR}(\mathrm{S}):$

Ida, Daichi

CITATION:

Ida, Daichi. Translational diffusion coefficient of wormlike regular threearm stars. Polymer Journal 2015, 47(10): 679-685

ISSUE DATE:

2015-06-17

URL:

http://hdl.handle.net/2433/202558

\section{RIGHT:}

(c) 2015 The Society of Polymer Science, Japan; This is the accepted manuscript of an article is available at http://dx.doi.org/10.1038/pj.2015.44; The full-text file will be made open to the public on 17 December 2015 in accordance with publisher's 'Terms and Conditions for Self-Archiving'.; This is not the published version. Please cite only the published version.; この論文は出版社版でありません。引用の際には出版社版をご確認ご利用ください。 
Translational diffusion coefficient of wormlike regular three-arm stars

Daichi Ida

Department of Polymer Chemistry, Kyoto University, Kyoto, Japan

Correspondence: Dr. D. Ida, Department of Polymer Chemistry, Kyoto University, Katsura, Kyoto 615-8510, Japan.

E-mail: ida@molsci.polym.kyoto-u.ac.jp 
ABSTRACT: Effects of chain stiffness on the translational diffusion coefficient $D$ or (effective) hydrodynamic radius $R_{\mathrm{H}}\left(\propto D^{-1}\right)$ are examined theoretically for the regular three-arm star polymers on the basis of the Kratky-Porod (KP) wormlike chain model. The ratio $g_{\mathrm{H}}$ of $R_{\mathrm{H}}$ of the regular KP three-arm star touched-bead model to that of the KP linear one, both having the same (reduced) total contour length $L$ and (reduced) bead diameter $d_{\mathrm{b}}$, is numerically evaluated on the basis of the Kirkwood formula and/or the Kirkwood-Riseman (KR) hydrodynamic equation. From an examination of the behavior of the Kirkwood value $g_{\mathrm{H}}^{(\mathrm{K})}$ and the KR one $g_{\mathrm{H}}^{(\mathrm{KR})}$ of $g_{\mathrm{H}}$ as a function of $L$ and $d_{\mathrm{b}}$, it is found that both of $g_{\mathrm{H}}^{(\mathrm{K})}$ and $g_{\mathrm{H}}^{(\mathrm{KR})}$ are insensitive to change in $L$ irrespective of the value of $d_{\mathrm{b}}$ and that $g_{\mathrm{H}}^{(\mathrm{KR})}$ is slightly larger than $g_{\mathrm{H}}^{(\mathrm{K})}$ in the ranges of $L$ and $d_{\mathrm{b}}$ investigated. An empirical interpolation formula is constructed for $g_{\mathrm{H}}^{(\mathrm{K})}$, which reproduces the asymptotic values $\sqrt{3} /(2 \sqrt{2}-1)(=0.947)$ in the random-coil limit and 1 in the thin rod limit.

KEYWORDS: hydrodynamic radius, semiflexible polymer; star polymers translational diffusion coefficient; wormlike chain model 


\section{INTRODUCTION}

We have made theoretical and/or computational studies of the intrinsic viscosity $[\eta]^{1,2}$ and second virial coefficient $A_{2}{ }^{3}$ of the semiflexible regular three-arm stars. The quantities $[\eta]$ and $A_{2}$ are measures of the average chain dimension as well as the mean-square radius of gyration $\left\langle S^{2}\right\rangle$, although $A_{2}$ is related to the chain dimension only in good solvents or perturbed state. The ratio $g_{\eta}$ of $[\eta]$ of an unperturbed regular three-arm star chain to that of the corresponding unperturbed linear one, both having the same molecular weight and chain stiffness, has been shown to become remarkably smaller than the random-coil limiting value as the chains become stiffer, as in the case of the ratio $g_{S}$ of $\left\langle S^{2}\right\rangle$ of the former chain to that of the latter. ${ }^{4}$ Further, for practical purposes, an empirical interpolation formula for $g_{\eta}$ has been constructed. On the other hand, the ratio $g_{A_{2}}$ of $A_{2}$ of a perturbed regular three-arm star chain to that of the corresponding perturbed linear one has been shown to be rather insensitive to change in chain stiffness.

The (effective) hydrodynamic radius $R_{\mathrm{H}}$ is another measure of the chain dimension and is defined from the translational diffusion coefficient $D$ as follows,

$$
R_{\mathrm{H}}=k_{\mathrm{B}} T / 6 \pi \eta_{0} D
$$

where $k_{\mathrm{B}}$ is the Boltzmann constant, $T$ is the absolute temperature, and $\eta_{0}$ is the viscosity coefficient of the solvent. It is then interesting and necessary to examine the effects of chain stiffness on the ratio $g_{\mathrm{H}}$ of $R_{\mathrm{H}}$ of the regular three-arm star chain to that of the corresponding linear one. In this paper, we evaluate $g_{\mathrm{H}}$ of the semiflexible regular three-arm star polymer on the basis of the KP chain without excluded volume as in the cases of the previous study of $g_{\eta}{ }^{2}$ and then, we construct an interpolation formula for $g_{\mathrm{H}}$ for practical purposes.

For an evaluation of $D$ for both of the KP regular three-arm star and linear chains, we adopt the touched-bead hydrodynamic model as in the case of the previous study of $[\eta] .^{2}$ And also, we use the Kirkwood formula ${ }^{5-7}$ as in the case of the linear helical wormlike touched-bead model $^{8,9}$ including the KP chain as a special case. On the basis of the Kirkwood formula, for 
unperturbed linear chains in the random-coil limit, there holds the asymptotic relation,

$$
D=0.196 k_{\mathrm{B}} T / \eta_{0}\left\langle R^{2}\right\rangle^{1 / 2} \quad \text { (linear, Kirkwood) }
$$

where $\left\langle R^{2}\right\rangle$ is the mean-square end-to-end distance of the chains. On the other hand, if $D$ is evaluated from the Kirkwood-Riseman (KR) hydrodynamic equation in the scheme of preaveraged hydrodynamic interaction $(\mathrm{HI}),{ }^{7,10}$ we have another asymptotic relation given by

$$
D=0.192 k_{\mathrm{B}} T / \eta_{0}\left\langle R^{2}\right\rangle^{1 / 2} \quad(\text { linear, KR) }
$$

Note that the correct numerical factor 0.192 of Eq. (3) was obtained by Kurata and Yamakawa $^{11}$ instead of the original approximate one 0.196 obtained in the so-called KR approximation for $D .^{7,10}$ We also note that the Zimm theory ${ }^{12}$ on the basis of the unperturbed (dynamic) Gaussian spring-bead model in the scheme of preaveraged HI gives the latter relation. The difference between the prefactor of the right-hand side of Eq. (2) and that of Eq. (3) is arising from the fact that the Kirkwood and KR (or Zimm) values of $D$ correspond to the translational diffusion of the center of mass of the polymer chain and that of the "Zimm center of resistance" 7,12 as defined as the point where the translational motion of the entire chain becomes independent of the internal (segmental) motions in the scheme of preaveraged HI, respectively. ${ }^{8,13,14}$ This may be expected to be the case with the star polymers. We then also evaluate $D$ (or $R_{\mathrm{H}}$ ) and $g_{\mathrm{H}}$ for the KP regular three-arm star chain following the KR procedure and examine difference between the Kirkwood and KR values of $R_{\mathrm{H}}$ and $g_{\mathrm{H}}$. This is another purpose of this paper.

\section{MODEL AND METHOD}

The model used in this study is the same as that used in the previous one, ${ }^{2}$ i.e., a regular threearm star touched-bead model composed of $3 m+1$ identical spherical beads of (hydrodynamic) diameter $d_{\mathrm{b}}$ whose centers are located on the KP regular three-arm star chain contour (see Figure 1 of Ref. 2). For convenience, the three arms are designated the first, second, and third ones and the $m$ beads on the $i$ th $(i=1,2,3)$ arm are numbered $(i-1) m+1,(i-1) m+2, \cdots$, $i m$ from the branch point (center) to the terminal end, with the center bead numbered 0 . The 
angle between each pair of the unit vectors tangent to the KP contours at the branch point is fixed to be $120^{\circ}$, so that the three vectors are on the same plane. The linear touched-bead model, the counterpart of the above star one, is the KP touched-bead model composed of $n+1$ identical beads of diameter $d_{\mathrm{b}}$ whose centers are located on the KP linear chain contour. We set $n+1$ equal to $3 m+1$, so that $n=3 m$. The $n+1$ beads are numbered $0,1,2, \cdots$, $n$ from one end to the other. For both the star and linear touched-bead models, the contour distance between the two adjacent beads is set equal to $d_{\mathrm{b}}$. In what follows, all lengths are measured in units of the stiffness parameter $\lambda^{-1}$ of the KP chain unless otherwise specified.

\section{Kirkwood formula}

The Kirkwood formula for $D$ of the chain composed of $n+1$ beads may be given by ${ }^{5-7}$

$$
D=\frac{k_{\mathrm{B}} T}{(n+1) \zeta}\left[1+\frac{\zeta}{6 \pi \eta_{0}(n+1)} \sum_{\substack{i=0 \\ i \neq j}}^{n} \sum_{\substack{j=0 \\ i}}^{n}\left\langle R_{i j}^{-1}\right\rangle\right] .
$$

where $\zeta=3 \pi \eta_{0} d_{\mathrm{b}}$ is the translational friction coefficient of bead and $\left\langle R_{i j}^{-1}\right\rangle$ is the mean reciprocal of the distance between the centers of the $i$ th and $j$ th beads.

For the $[(i-1) m+k]$ th and $[(j-1) m+l]$ th beads $(i, j=1,2,3 ; k, l=1,2, \cdots, m)$ of the KP regular three-arm star chain, i.e., the $k$ th bead on the $i$ th arm and the $l$ th bead on the $j$ th arm, respectively, $\left\langle R_{[(i-1) m+k][(j-1) m+l]}{ }^{-1}\right\rangle$ may be given by

$$
\left\langle R_{[(i-1) m+k][(j-1) m+l]}{ }^{-1}\right\rangle=\left\langle R^{-1}\left(t_{k}^{(i)}, t_{l}^{(j)}\right)\right\rangle,
$$

with $t_{k}^{(i)}$ denoting the contour distance from the branch point to the contour point on the $i$ th arm where the center of the $[(i-1) m+k]$ th bead locates on, so that

$$
t_{k}^{(i)}=k d_{\mathrm{b}}
$$

The theoretical expression for $\left\langle R^{-1}\left(t_{k}^{(i)}, t_{l}^{(j)}\right)\right\rangle$ has been obtained in the previous paper. ${ }^{2}$ Here we give only the results with a brief description. For the KP regular three-arm star chain under the consideration, $\left\langle R^{-1}\left(t_{k}^{(i)}, t_{l}^{(j)}\right)\right\rangle$ may be given by

$$
\begin{aligned}
\left\langle R^{-1}\left(t_{k}^{(i)}, t_{l}^{(j)}\right)\right\rangle & =\left\langle R^{-1}\left(t_{k}^{(i)}, t_{l}^{(j)}, 120^{\circ}\right)\right\rangle & & \text { for } i \neq j \\
& =\left\langle R^{-1}\left(\left|t_{k}^{(i)}-t_{l}^{(i)}\right|, 0,120^{\circ}\right)\right\rangle & & \text { for } i=j,
\end{aligned}
$$


where $\left\langle R^{-1}\left(t_{1}, t_{2}, 120^{\circ}\right)\right\rangle$ is the mean reciprocal of the end-to-end distance of the (unperturbed) once-broken KP chain of total contour length $t_{1}+t_{2}$ such that two KP subchains 1 and 2 of contour lengths $t_{1}$ and $t_{2}$, respectively, are connected with a bending angle $\theta=120^{\circ}$ (see Figure 2 in Ref. 2). We note that $\left\langle R^{-1}\left(t, 0,120^{\circ}\right)\right\rangle$ and/or $\left\langle R^{-1}\left(0, t, 120^{\circ}\right)\right\rangle$ represent the mean reciprocal of the end-to-end distance of the KP linear chain of contour length $t$. The interpolation formula for $\left\langle R^{-1}\left(t_{1}, t_{2}, 120^{\circ}\right)\right\rangle$ may be given by

$$
\begin{aligned}
\left\langle R^{-1}\left(t_{1}, t_{2}, 120^{\circ}\right)\right\rangle= & \left\langle R^{2}\right\rangle^{-1 / 2}\left[1+5\left\langle R^{2}\right\rangle^{11 / 2}\right]^{-1}\left[f_{\epsilon \epsilon}\left(t_{1}, t_{2}, 120^{\circ}\right)+0.045\left\langle R^{2}\right\rangle^{3 / 2}\right. \\
& \left.+0.40 t_{1} t_{2}\left(t_{1}+t_{2}\right) e^{-t_{1} t_{2}}+5\left\langle R^{2}\right\rangle^{11 / 2} f_{\mathrm{DD} \epsilon}\left(t_{1}, t_{2}, 120^{\circ}\right)\right]
\end{aligned}
$$

where

$$
\begin{gathered}
f_{\epsilon \epsilon}\left(t_{1}, t_{2}, \theta\right)=1+\frac{3}{8}\left(\frac{\left\langle R^{4}\right\rangle}{\left\langle R^{2}\right\rangle^{2}}-1\right), \\
f_{\mathrm{DD} \epsilon}\left(t_{1}, t_{2}, 120^{\circ}\right)=\left(\frac{6}{\pi}\right)^{1 / 2}\left[1-\frac{11}{40\left\langle R^{2}\right\rangle}+\frac{431}{4480\left\langle R^{2}\right\rangle^{2}}+\frac{C\left(t_{1}, t_{2}\right)}{\left\langle R^{2}\right\rangle^{2}}\right],
\end{gathered}
$$

and $C\left(t_{1}, t_{2}\right)$ is given by

$$
C\left(t_{1}, t_{2}\right)=\frac{108 \bar{t}^{2}+77 \bar{t}^{4}}{640\left(1+\bar{t}^{4}\right)}
$$

with

$$
\vec{t}^{2}=\left(t_{1}^{-2}+t_{2}^{-2}\right)^{-1}
$$

In Eqs. (8)-(10), $\left\langle R^{2}\right\rangle=\left\langle R^{2}\left(t_{1}, t_{2}, \theta\right)\right\rangle$ and $\left\langle R^{4}\right\rangle=\left\langle R^{4}\left(t_{1}, t_{2}, \theta\right)\right\rangle$ are the second and fourth moments, respectively, of the end-to-end distance of the once-broken KP chain are given by

$$
\begin{aligned}
& \left\langle R^{2}\left(t_{1}, t_{2}, \theta\right)\right\rangle=t_{1}+t_{2}-\frac{1}{2}\left[1-e^{-2\left(t_{1}+t_{2}\right)}\right]-\frac{1}{2}\left(1-e^{-2 t_{1}}\right)\left(1-e^{-2 t_{2}}\right)(1+\cos \theta), \\
& \left\langle R^{4}\left(t_{1}, t_{2}, \theta\right)\right\rangle \\
& =\frac{5}{3}\left(t_{1}+t_{2}\right)^{2}-\left(t_{1}+t_{2}\right)\left[\frac{26}{9}+e^{-2\left(t_{1}+t_{2}\right)}\right]+2\left[1-e^{-2\left(t_{1}+t_{2}\right)}\right]-\frac{1}{54}\left[1-e^{-6\left(t_{1}+t_{2}\right)}\right] \\
& \quad-\left\{\left(1-e^{-2 t_{1}}\right)\left[\frac{5}{3} t_{2}+t_{2} e^{-2 t_{2}}-\frac{3}{2}\left(1-e^{-2 t_{2}}\right)+\frac{1}{18}\left(1-e^{-6 t_{2}}\right)\right]\right. \\
& \left.\quad+\left(1-e^{-2 t_{2}}\right)\left[\frac{5}{3} t_{1}+t_{1} e^{-2 t_{1}}-\frac{3}{2}\left(1-e^{-2 t_{1}}\right)+\frac{1}{18}\left(1-e^{-6 t_{1}}\right)\right]\right\}(1+\cos \theta) \\
& \quad-\frac{1}{4}\left[\left(1-e^{-2 t_{1}}\right)-\frac{1}{3}\left(1-e^{-6 t_{1}}\right)\right]\left[\left(1-e^{-2 t_{2}}\right)-\frac{1}{3}\left(1-e^{-6 t_{2}}\right)\right]\left(1-\cos ^{2} \theta\right) .
\end{aligned}
$$


We note that the expression for $\left\langle R^{2}\left(t_{1}, t_{2}, \theta\right)\right\rangle$ given by Eq. (13) was first derived by MansfieldStockmayer. ${ }^{4}$ In the rod limit, Eq. (8) reduces to

$$
\left.\left\langle R^{-1}\left(t_{1}, t_{2}, 120^{\circ}\right)\right\rangle=\left(t_{1}^{2}+t_{2}^{2}+t_{1} t_{2}\right)^{-1 / 2} \quad \text { (rod limit }\right)
$$

For later convenience, $D$ obtained from Eq. (2) is designated $D^{(\mathrm{K})}$ hereafter.

\section{Kirkwood-Riseman equation}

On the basis of the KR hydrodynamic equation in the scheme of preaveraged $\mathrm{HI},{ }^{7,10} \mathrm{D}$ of the chain composed of $n+1$ beads may be written as follows,

$$
D=k_{\mathrm{B}} T / \zeta \sum_{i=0}^{n} \psi_{i},
$$

where $\psi_{i}$ is the solution of the following linear simultaneous equations,

$$
\psi_{i}=1-\frac{\zeta}{6 \pi \eta_{0}} \sum_{\substack{j=0 \\ \neq i}}^{n}\left\langle R_{i j}{ }^{-1}\right\rangle \psi_{j} .
$$

The expression for $\left\langle R_{i j}^{-1}\right\rangle$ has already been given by Eq. (5) with Eqs. (7)-(14). We note that if we assume $\psi_{i}=(n+1)^{-1} \psi$, i.e., the average force exerted on the solvent of the $i$ th bead equal to the average total force of the entire chain divided by $n+1$, for all $i$, Eqs. (16) and (17) may reduce to Eq. (4). We also note that this assumption is equivalent to the KR approximation for $D$ mentioned in INTRODUCTION. For later convenience, $D$ obtained from Eqs. (16) and (17) is designated $D^{(\mathrm{KR})}$ hereafter.

\section{RESULTS AND DISCUSSION}

We have calculated the Kirkwood value $D^{(\mathrm{K})}$ and the $\mathrm{KR}$ one $D^{(\mathrm{KR})}$ of the translational diffusion coefficient $D$ from Eq. (4) and from Eq. (16) with the numerical solution $\psi_{i}$ of the linear simultaneous equations 17, respectively, for both the KP regular three-arm star and linear touched-bead models, in the ranges of the total number $n+1$ of bonds from 4 to 9001 and of the bead diameter $d_{\mathrm{b}}$ from 0.001 to 0.4 . Note that the total contour length $L$ of the chain is equal to $(n+1) d_{\mathrm{b}}$, as already mentioned in MODEL AND METHOD. In Eqs. (4) and (17), $\left\langle R_{i j}^{-1}\right\rangle$ is given by Eq. (5) with Eqs.(7)-(14). On the basis of the values of $D^{(\mathrm{K})}$ and 
$D^{(\mathrm{KR})}$ for the star and linear chains having the same $L$ and $d_{\mathrm{b}}$ so obtained along with Eq. (1), we evaluate the Kirkwood value $g_{\mathrm{H}}^{(\mathrm{K})}$ and the KR one $g_{\mathrm{H}}^{(\mathrm{KR})}$ of the ratio $g_{\mathrm{H}}$ as functions of $L$ and $d_{\mathrm{b}}$ defined by

$$
g_{\mathrm{H}}^{(\mathrm{K})}\left(L, d_{\mathrm{b}}\right)=\frac{R_{\mathrm{H}}^{(\mathrm{K})}(\text { star })}{R_{\mathrm{H}}^{(\mathrm{K})}(\text { linear })}=\frac{D^{(\mathrm{K})}(\text { linear })}{D^{(\mathrm{K})}(\text { star })}
$$

and

$$
g_{\mathrm{H}}^{(\mathrm{KR})}\left(L, d_{\mathrm{b}}\right)=\frac{R_{\mathrm{H}}^{(\mathrm{KR})}(\text { star })}{R_{\mathrm{H}}^{(\mathrm{KR})}(\text { linear })}=\frac{D^{(\mathrm{KR})}(\text { linear })}{D^{(\mathrm{KR})}(\mathrm{star})},
$$

respectively.

In the following subsections, we first examine the behavior of $g_{\mathrm{H}}^{(\mathrm{K})}$ and $g_{\mathrm{H}}^{(\mathrm{KR})}$ as functions of $L$ and $d_{\mathrm{b}}$ and compare the theoretical values of the two ratios. Then we construct an interpolation formula for $g_{\mathrm{H}}^{(\mathrm{K})}$.

\section{Figure 1}

\section{Comparison between $g_{\mathrm{H}}^{(\mathrm{K})}$ and $g_{\mathrm{H}}^{(\mathrm{KR})}$}

Figure 1 shows plots of $g_{\mathrm{H}}$ against the logarithm of $L$. The open and closed circles represent the theoretical values of $g_{\mathrm{H}}^{(\mathrm{K})}$ and $g_{\mathrm{H}}^{(\mathrm{KR})}$, respectively, for $d_{\mathrm{b}}=0.001$ (pip up), 0.003 (pip rightup), 0.01 (pip right), 0.03 (pip right-down), 0.1 (pip down), 0.2 (pip left-down), 0.3 (pip left), and 0.4 (pip left-up), the dashed curves connecting smoothly the respective theoretical values at constant $d_{\mathrm{b}}$. The solid curves represent the values calculated from an interpolation formula for $g_{\mathrm{H}}^{(\mathrm{K})}$, as discussed later.

In the case of $g_{\mathrm{H}}^{(\mathrm{K})}$, the asymptotic value in the random-coil limit, i.e., the limit $L \rightarrow \infty$ (in units of $\lambda^{-1}$ ) may be given by

$$
\lim _{L \rightarrow \infty} g_{\mathrm{H}}^{(\mathrm{K})}\left(L, d_{\mathrm{b}}\right)=\sqrt{3} /(2 \sqrt{2}-1)=0.947 \quad(\text { random coil })
$$

which may be calculated from the relation $g_{\mathrm{H}}^{(\mathrm{K})}=\left[(\sqrt{2}-1) f^{1 / 2}+(2-\sqrt{2}) f^{-1 / 2}\right]^{-1}$ obtained for the Gaussian regular $f$-arm stars by Kurata and Fukatsu ${ }^{15}$ and by Stockmayer and Fixman ${ }^{16}$ (, the latter authors using the $\mathrm{KR}$ approximation for $D$ ). The asymptotic value is represented by the lower horizontal line. As $L$ is decreased, $g_{\mathrm{H}}^{(\mathrm{K})}$ first increases from the random-coil limiting value and then decreases and exhibits a minimum after passing through a maximum, 
in the range of $d_{\mathrm{b}}$ investigated except for $d_{\mathrm{b}}=0.4$. The behavior of $g_{\mathrm{H}}^{(\mathrm{K})}$ depends also on $d_{\mathrm{b}}$. It should be noted that the difference between the maximum and minimum of $g_{\mathrm{H}}^{(\mathrm{K})}$ is rather small (4\% at most).

\section{Figure 2}

As for $g_{\mathrm{H}}^{(\mathrm{KR})}$, its values are slightly $\left(3 \%\right.$ at most) larger than those of $g_{\mathrm{H}}^{(\mathrm{K})}$ and exhibit no appreciable maximum in contrast to the case of $g_{\mathrm{H}}^{(\mathrm{K})}$, in the ranges of $L$ and $d_{\mathrm{b}}$ investigated. We have evaluated the random-coil limiting value of $g_{\mathrm{H}}^{(\mathrm{KR})}\left(L, d_{\mathrm{b}}\right)$ from the numerical theoretical values with large $d_{\mathrm{b}}$. Figure 2 shows plots of $g_{\mathrm{H}}^{(\mathrm{KR})}$ against $L^{-1 / 2}$ for $d_{\mathrm{b}}=0.1,0.2,0.3$, and 0.4. All the symbols have the same meaning as those in Fig. 1. The dashed curves connect smoothly the theoretical values at constant $d_{\mathrm{b}}$ and solid lines indicate the respective initial tangents. It is seen that as $L^{-1 / 2}$ is decreased to $0(L \rightarrow \infty), g_{\mathrm{H}}^{(\mathrm{KR})}$ approaches a constant value irrespective of the value of $d_{\mathrm{b}}$. On the basis of such numerical results, it may be concluded that

$$
\lim _{L \rightarrow \infty} g_{\mathrm{H}}^{(\mathrm{KR})}\left(L, d_{\mathrm{b}}\right)=0.96_{4} \quad(\text { random coil }) .
$$

Note that the values of $g_{\mathrm{H}}^{(\mathrm{KR})}$ for smaller $d_{\mathrm{b}}$ have been omitted in Fig. 2, since we cannot make $L^{-1 / 2}\left(=\left[(n+1) d_{\mathrm{b}}\right]^{-1 / 2}\right)$ small enough to evaluate $g_{\mathrm{H}}^{(\mathrm{KR})}$ at $L^{-1 / 2}=0$. We also note that the asymptotic value so obtained is consistent with an available theoretical value of 0.96 obtained by Irurzun ${ }^{17}$ for the Gaussian regular three-arm star chain without excluded volume on the basis of the KR equation. In Fig. 1, the upper horizontal line represents the asymptotic value 0.964 . This asymptotic value is $1.8 \%$ larger than that of $g_{\mathrm{H}}^{(\mathrm{K})}$ given by Eq. (20).

For an examination the difference between $g_{\mathrm{H}}^{(\mathrm{K})}$ and $g_{\mathrm{H}}^{(\mathrm{KR})}$, it is useful to derive the asymptotic relations between $R_{\mathrm{H}}$ and $\left\langle S^{2}\right\rangle^{1 / 2}$ in the random-coil limit for both of the regular threearm star and linear chains. From Eqs. (2) and (3) using the asymptotic relation $\left\langle R^{2}\right\rangle=6\left\langle S^{2}\right\rangle$ for the linear chain ${ }^{7}$ in this limit along with Eq. (1), we may obtain

$$
\begin{gathered}
R_{\mathrm{H}}^{(\mathrm{K})}=0.663\left\langle S^{2}\right\rangle^{1 / 2} \quad \text { (random coil, linear) } \\
R_{\mathrm{H}}^{(\mathrm{KR})}=0.677\left\langle S^{2}\right\rangle^{1 / 2} \quad \text { (random coil, linear) } .
\end{gathered}
$$


As for the regular three-arm star chain in the random-coil limit, from Eqs. (18)-(23) using with the asymptotic value $7 / 9$ of $g_{S},{ }^{18}$ we then obtain the relations

$$
\begin{gathered}
R_{\mathrm{H}}^{(\mathrm{K})}=0.712\left\langle S^{2}\right\rangle^{1 / 2} \quad \text { (random coil, star) } \\
R_{\mathrm{H}}^{(\mathrm{KR})}=0.740\left\langle S^{2}\right\rangle^{1 / 2} \quad \text { (random coil, star) } .
\end{gathered}
$$

It is seen that for the regular three-arm star chain $R_{\mathrm{H}}^{(\mathrm{KR})}$ is $3.8 \%$ larger than $R_{\mathrm{H}}^{(\mathrm{K})}$, while for the linear chain the former value is $2.1 \%$ larger than the latter.

Further we give the asymptotic forms of $g_{\mathrm{H}}^{(\mathrm{K})}$ and $g_{\mathrm{H}}^{(\mathrm{KR})}$ in the thin rod limit, i.e., the limit of $L \rightarrow 0$ (in units of $\lambda^{-1}$ ) and $L / d_{\mathrm{b}} \rightarrow \infty$. In this limit, $D^{(\mathrm{K})}$ for the regular three-ram star chain may be written in the form (see Appendix),

$$
\lim _{\substack{L \rightarrow 0 \\ L / d_{\mathrm{b}} \rightarrow \infty}} D^{(\mathrm{K})}=\frac{k_{\mathrm{B}} T \ln \left(L / d_{\mathrm{b}}\right)}{3 \pi \eta_{0} L} \quad \text { (thin rod limit, star). }
$$

As for the linear chain, we have ${ }^{7}$

$$
\lim _{\substack{L \rightarrow 0 \\ L / d_{\mathrm{b}} \rightarrow \infty}} D^{(\mathrm{K})}=\frac{k_{\mathrm{B}} T \ln \left(L / d_{\mathrm{b}}\right)}{3 \pi \eta_{0} L} \quad \text { (thin rod limit, linear). }
$$

We note that Eq. (27) may be obtained straightforwardly from Eq. (4) with Eqs. (5), (7), and (15) along with the relation $L=(n+1) d_{\mathrm{b}}$. In the rod limit, $g_{\mathrm{H}}^{(\mathrm{K})}$ should be a function only of $L / d_{\mathrm{b}}$, i.e.,

$$
\lim _{L \rightarrow 0} g_{\mathrm{H}}^{(\mathrm{K})}\left(L, d_{\mathrm{b}}\right)=g_{\mathrm{H}, 0}^{(\mathrm{K})}\left(L / d_{\mathrm{b}}\right) \quad(\text { rod limit }) .
$$

From Eq. (18) with Eqs. (26), (27), and (28), we have

$$
\lim _{L / d_{\mathrm{b}} \rightarrow \infty} g_{\mathrm{H}, 0}^{(\mathrm{K})}\left(L / d_{\mathrm{b}}\right)=1 \quad \text { (thin rod limit) } .
$$

On the other hand, $D^{(\mathrm{KR})}$ for the regular three-arm star chain (see Appendix) and that for the linear one ${ }^{19}$ in the thin rod limit may be written in the forms,

$$
\lim _{\substack{L \rightarrow 0 \\ L / d_{\mathrm{b}} \rightarrow \infty}} D^{(\mathrm{KR})}=\frac{k_{\mathrm{B}} T \ln \left(L / d_{\mathrm{b}}\right)}{3 \pi \eta_{0} L} \quad \text { (thin rod limit, star) }
$$

and

$$
\lim _{\substack{L \rightarrow 0 \\ L / d_{\mathrm{b}} \rightarrow \infty}} D^{(\mathrm{KR})}=\frac{k_{\mathrm{B}} T \ln \left(L / d_{\mathrm{b}}\right)}{3 \pi \eta_{0} L} \quad \text { (thin rod limit, linear) }
$$


respectively. In the rod limit, $g_{\mathrm{H}}^{(\mathrm{KR})}$ should also be a function only of $L / d_{\mathrm{b}}$, i.e.,

$$
\lim _{L \rightarrow 0} g_{\mathrm{H}}^{(\mathrm{KR})}\left(L, d_{\mathrm{b}}\right)=g_{\mathrm{H}, 0}^{(\mathrm{KR})}\left(L / d_{\mathrm{b}}\right) \quad(\text { rod limit })
$$

From Eq. (19) with Eqs. (30), (31), and (32), we have

$$
\lim _{L / d_{\mathrm{b}} \rightarrow \infty} g_{\mathrm{H}, 0}^{(\mathrm{KR})}\left(L / d_{\mathrm{b}}\right)=1 \quad \text { (thin rod limit) } .
$$

All of these equations for $D^{(\mathrm{KR})}$ and $g_{\mathrm{H}}^{(\mathrm{KR})}$ have the same forms as the corresponding ones for $D^{(\mathrm{K})}$ and $g_{\mathrm{H}}^{(\mathrm{K})}$ given by Eqs. (26), (27), and (29).

Such salient results for given by Eqs. (29) or (33) that the translational diffusion coefficient of the regular three-arm star chain becomes identical with that of the corresponding linear chain in the thin rod limit may be regarded as indicating the defect of the Kirkwood formula or that of the scheme of preaveraged $\mathrm{HI}$ as in the case of rigid rings. ${ }^{7,8,20-24}$

\section{Interpolation formula for $g_{\mathrm{H}}^{(\mathrm{K})}$}

Now we are in a position to construct an interpolation formula for $g_{\mathrm{H}}^{(\mathrm{K})}$ on the basis of the numerical theoretical values of $g_{\mathrm{H}}^{(\mathrm{K})}\left(L, d_{\mathrm{b}}\right)$ as already shown in Fig. 1 along with the asymptotic relations given by Eqs. (20) and (29) in the random coil and thin rod limits, respectively.

\section{Figure 3}

We have evaluated $g_{\mathrm{H}, 0}^{(\mathrm{K})}\left(L / d_{\mathrm{b}}\right)$ numerically in the same manner as in the evaluation of $g_{\mathrm{H}}^{(\mathrm{K})}\left(L, d_{\mathrm{b}}\right)$ mentioned above using the expression $\left\langle R_{i j}^{-1}\right\rangle$ given by Eqs. (5), (7), and (15) in place of that for the KP chain. Figure 3 shows plots of $g_{\mathrm{H}, 0}^{(\mathrm{K})}$ against $\left[\ln \left(L / d_{\mathrm{b}}\right)\right]^{-1}$. The open circles represent the values so obtained. As $\left[\ln \left(L / d_{\mathrm{b}}\right)\right]^{-1}$ is decreased $\left(L / d_{\mathrm{b}}\right.$ is increased $), g_{\mathrm{H}, 0}^{(\mathrm{K})}$ first decreases and then increases to the asymptotic value 1 after passing through a minimum. For later convenience,we have constructed an interpolation formula for $g_{\mathrm{H}, 0}^{(\mathrm{K})}\left(L / d_{\mathrm{b}}\right)$ in the range of $L / d_{\mathrm{b}} \gtrsim 10$, which is given by

$$
g_{\mathrm{H}, 0}^{(\mathrm{K})}(x)=\frac{1-1.84723(\ln x)^{-1}+9.01492(\ln x)^{-2}}{1-1.46180(\ln x)^{-1}+8.88140(\ln x)^{-2}} \quad \text { for } x \gtrsim 10 .
$$

In Fig. 3, the curve represents the values calculated from Eq. (34) with $x=L / d_{\mathrm{b}}$. The error in the value of $g_{\mathrm{H}, 0}^{(\mathrm{K})}\left(L / d_{\mathrm{b}}\right)$ in the range of $L / d_{\mathrm{b}} \gtrsim 10$ (solid part) does not exceed $0.1 \%$. 


\section{Figure 4}

Next, we consider the ratio $g_{\mathrm{H}}^{(\mathrm{K})} / g_{\mathrm{H}, 0}^{(\mathrm{K})}$. Figure 4 shows plots of $g_{\mathrm{H}}^{(\mathrm{K})}\left(L, d_{\mathrm{b}}\right) / g_{\mathrm{H}, 0}^{(\mathrm{K})}\left(L / d_{\mathrm{b}}\right)$ against the logarithm of $L$, where $g_{\mathrm{H}}^{(\mathrm{K})} / g_{\mathrm{H}, 0}^{(\mathrm{K})}$ has been evaluated by dividing the $g_{\mathrm{H}}^{(\mathrm{K})}$ values shown in Fig. 1 by the $g_{\mathrm{H}, 0}^{(\mathrm{K})}$ values calculated from Eq. (34) with $x=L / d_{\mathrm{b}}$. All the symbols in Fig. 4 have the same meaning as those in Fig. 1 . It is seen that as $L$ is increased, $g_{\mathrm{H}}^{(\mathrm{K})} / g_{\mathrm{H}, 0}^{(\mathrm{K})}$ as a function of $L$ and $d_{\mathrm{b}}$, which is represented by $f\left(L, d_{\mathrm{b}}\right)$ hereafter, first increases from unity and then decreases after passing through a maximum in the range of $d_{\mathrm{b}}$ investigated. Considering the asymptotic conditions $\lim _{L \rightarrow 0} f\left(L, d_{\mathrm{b}}\right)=1$ and $\lim _{L \rightarrow \infty} f\left(L, d_{\mathrm{b}}\right)=\sqrt{3} /(2 \sqrt{2}-1)$, which hold in the limit of $L / d_{\mathrm{b}} \rightarrow \infty$, we have constructed an interpolation formula for $f\left(L, d_{\mathrm{b}}\right)$, which may be written in the form,

$$
\begin{aligned}
f\left(L, d_{\mathrm{b}}\right) & =1+a_{1} L+a_{2} L^{2} & & \text { for } L<6 \\
& =\left[b_{0}-1+b_{1}(\ln L)^{-1}\right] \exp \left[-b_{2}(\ln L)^{-1}-b_{3}(\ln L)^{-2}\right]+1 & & \text { for } L \geq 6 .
\end{aligned}
$$

In Eq. (35), the coefficients $a_{i}(i=1,2)$ and $b_{i}(i=0,1,2,3)$ may be given by

$$
\begin{aligned}
& a_{1}=\left[f\left(6, d_{\mathrm{b}}\right)-3 f^{\prime}\left(6, d_{\mathrm{b}}\right)-1\right] / 3 \\
& a_{2}=\left[-f\left(6, d_{\mathrm{b}}\right)+6 f^{\prime}\left(6, d_{\mathrm{b}}\right)+1\right] / 36
\end{aligned}
$$

and

$$
\begin{aligned}
& b_{0}=\sqrt{3} /(2 \sqrt{2}-1) \\
& b_{1}=0.276181+0.473221 d_{\mathrm{b}}-0.240008 d_{\mathrm{b}}^{2}+\left(0.018263+0.572154 d_{\mathrm{b}}^{2}\right) \ln d_{\mathrm{b}} \\
& b_{2}=2.66405-7.02277 d_{\mathrm{b}}+2.26380 d_{\mathrm{b}}{ }^{2}-\left(0.11568+7.14996 d_{\mathrm{b}}{ }^{2}\right) \ln d_{\mathrm{b}} \\
& b_{3}=4.50327+0.06635 d_{\mathrm{b}}+4.03912{d_{\mathrm{b}}}^{2}+\left(0.33442-9.70377 d_{\mathrm{b}}{ }^{2}\right) \ln d_{\mathrm{b}}
\end{aligned}
$$

respectively, where $f^{\prime}\left(L, d_{\mathrm{b}}\right)=\partial f\left(L, d_{\mathrm{b}}\right) / \partial L$. Note that in Eq. (36) the values of $f\left(6, d_{\mathrm{b}}\right)$ and $f^{\prime}\left(6, d_{\mathrm{b}}\right)$ may be calculated from Eq. (35) with Eq. (37). In Fig. 4, the solid curves represent the values calculated from Eqs. (35) - (37) with the corresponding values of $d_{\mathrm{b}}$. It is seen that the interpolation formula may well reproduce the numerical theoretical values in the range 
of $L$ and $d_{\mathrm{b}}$ so examined, although for $0 \lesssim L \lesssim 1$ the numerical theoretical values seem to deviate downward slightly (up to $0.5 \%$ ) from the corresponding values of the interpolation formula. Such a slight deviation is within experimental error (1\% at least) in $D$ determined by conventional methods and then causes no significant error in a practical use of the present interpolation formula for analysis of experimental data.

The factor $g_{\mathrm{H}}^{(\mathrm{K})}\left(L, d_{\mathrm{b}}\right)$ may therefore be approximately expressed as

$$
g_{\mathrm{H}}^{(\mathrm{K})}\left(L, d_{\mathrm{b}}\right)=g_{\mathrm{H}, 0}^{(\mathrm{K})}\left(L / d_{\mathrm{b}}\right) f\left(L, d_{\mathrm{b}}\right)
$$

where $g_{\mathrm{H}, 0}^{(\mathrm{K})}\left(L / d_{\mathrm{b}}\right)$ and $f\left(L, d_{\mathrm{b}}\right)$ are given by Eq. (34) and Eq. (35) with Eqs. (36) and (37), respectively. In Fig. 1, the solid curves represent the approximate values calculated from Eq. (38) with Eqs. (34)-(37) with the corresponding values of $d_{\mathrm{b}}$. It is seen that the interpolation formula for $g_{\mathrm{H}}^{(\mathrm{K})}\left(L, d_{\mathrm{b}}\right)$ so proposed may well reproduce the numerical theoretical values in the ranges of $d_{\mathrm{b}}$ investigated and of $L / d_{\mathrm{b}} \gtrsim 10$. The error in the value of $g_{\mathrm{H}}^{(\mathrm{K})}$ in those ranges of $d_{\mathrm{b}}$ and $L / d_{\mathrm{b}}$ does not exceed $0.4 \%$.

\section{Figure 5}

\section{Comparison with experiment}

Finally, a comparison of the present theoretical result with experimental data in a literature. Figure 5 shows plots of $g_{\mathrm{H}}$ against the logarithm of the weight-average molecular weight $M_{\mathrm{w}}$ for the regular three-arm star polystyrene in cyclohexane at $34.5^{\circ} \mathrm{C}(\Theta)$ obtained by Huber et.al..$^{25}$ The open circles represent the experimental values. The curve represents the KP theory values of $g_{\mathrm{H}}^{(\mathrm{K})}\left(\lambda \hat{L}, \lambda \hat{d}_{\mathrm{b}}\right)$, where $\hat{L}$ and $\hat{d}_{\mathrm{b}}$ is the total contour length and bead diameter, respectively, of the KP regular three-arm star touched-bead model in real length units, calculated from Eq. (38) with Eqs. (34)-(37) along with the relation $\log M_{\mathrm{w}}=\log \lambda \hat{L}+\log \left(\lambda^{-1} M_{\mathrm{L}}\right)$ with $M_{\mathrm{L}}$ the molecular weight per unit contour length. The solid part of the curve indicates the range of $L / d_{\mathrm{b}} \gtrsim 10$. The necessary KP parameter values used in the calculation of the KP theory values are $\lambda^{-1}=20.0 \AA$ and $M_{\mathrm{L}}=39.0 \AA^{-1}$ determined by Norisuye and Fujita ${ }^{26}$ for (linear) atactic-polystyrene in cyclohexane at $34.5{ }^{\circ} \mathrm{C}(\Theta)$ and $\hat{d}_{\mathrm{b}}=9.9 \AA$ estimated from the 
relation $^{28} d=0.891 \hat{d}_{\mathrm{b}}$ with $d$ the hydrodynamic diameter of the KP cylinder model ${ }^{8}$ using with $d=8.8 \AA$ determined for the same system by Huber et.al. ${ }^{27}$ It is seen that the present theory may explain qualitatively the behavior of the experimental values, which increase with decreasing $M_{\mathrm{w}}$, although including the range of $L / d_{\mathrm{b}} \lesssim 10$.

\section{CONCLUSION}

We have evaluated the Kirkwood value $g_{\mathrm{H}}^{(\mathrm{K})}$ and the KR one $g_{\mathrm{H}}^{(\mathrm{KR})}$ of the ratio $g_{\mathrm{H}}$ of $R_{\mathrm{H}}$ of the unperturbed KP regular three-arm star touched-bead model to that of the KP linear one, both having the same (reduced) total contour length $L$ and (reduced) bead diameter $d_{\mathrm{b}}$. From an examination of the behavior of $g_{\mathrm{H}}^{(\mathrm{K})}$ and that of $g_{\mathrm{H}}^{(\mathrm{KR})}$ as functions of $L$ and $d_{\mathrm{b}}$, it is found that both of $g_{\mathrm{H}}^{(\mathrm{K})}$ and $g_{\mathrm{H}}^{(\mathrm{KR})}$ are insensitive to change in $L$ irrespective of the value of $d_{\mathrm{b}}$ and that $g_{\mathrm{H}}^{(\mathrm{KR})}$ is $3 \%$ at most larger than $g_{\mathrm{H}}^{(\mathrm{KR})}$ in the ranges of $L$ and $d_{\mathrm{b}}$. The empirical interpolation formula for $g_{\mathrm{H}}^{(\mathrm{K})}$ has been constructed, which reproduces the asymptotic values $\sqrt{3} /(2 \sqrt{2}-1)$ $(=0.947)$ in the random-coil limit and 1 in the thin rod limit. 


\section{(REFERENCES)}

1 Ida, D. \& Yoshizaki, T. A Monte Carlo study of the intrinsic viscosity of semiflexible regular three-arm star polymers Polym. J. 39, 1373-1382 (2007).

2 Ida, D., Nakamura, Y. \& Yoshizaki, T. Intrinsic viscosity of wormlike regular three-arm stars Polym. J. 40, 256-267 (2008).

3 Ida, D. \& Yoshizaki, T. A Monte Carlo study of the second virial coefficient of semiflexible regular three-arm star polymers Polym. J. 40, 1074-1080 (2008).

4 Mansfield, M. L. \& Stockmayer, W. H. Unperturbed dimensions of wormlike stars Macromolecules 13, 1713-1715, (1980).

5 Kirkwood, J. G. The statistical mechanical theory of irreversible processes in solutions of flexible macromolecules. Visco-elastic behavior Rec. Trav. Chim. 68, 649-660 (1949).

6 Kirkwood, J. G. The general theory of irreversible processes in solutions of macromolecules J. Polym. Sci. 12, 1-14 (1954).

7 Yamakawa, H. Modern Theory of Polymer Solutions (Harper \& Row, New York, 1971). Its electronic edition is available on-line at the URL: http://www.molsci.polym.kyotou.ac.jp/archives/redbook.pdf

8 Yamakawa, H., Helical Wormlike Chains in Polymer Solutions (Springer, Berlin, 1997).

9 Yamada, T., Yoshizaki, T. \& Yamakawa, H. Transport coefficients of helical wormlike chains. 5. Translational diffusion coefficient of the touched-bead model and its application to oligo- and polystyrenes Macromolecules 25, 377-383, (1992).

10 Kirkwood, J. G. \& Riseman, J. The intrinsic viscosities and diffusion constants of flexible macromolecules in solution. J. Chem. Phys. 16, 565-573 (1948).

11 Kurata, M. \& Yamakawa, H. Theory of dilute polymer solution. II. Osmotic pressure and frictional properties J. Chem. Phys. 29, 311-325 (1958). 
12 Zimm, B. H. Dynamics of polymer molecules in dilute solution: Viscoelasticity, flow birefringence and dielectric loss J. Chem. Phys. 24, 269-278 (1956).

13 Yamakawa, H. \& Yoshizaki, T. Dynamics of helical wormlike chains. I. Dynamic model and diffusion equation J. Chem. Phys. 75, 1016-1030 (1981).

14 Yamakawa, H. \& Yoshizaki, T. Dynamics of helical wormlike chains. XI. Translational diffusion with fluctuating hydrodynamic interaction J. Chem. Phys. 91, 7900-7911 (1989).

15 Kurata, M. \& Fukatsu, M. Unperturbed dimension and translational friction constant of branched polymers J. Chem. Phys. 41, 2934-2944 (1964).

16 Stockmayer, W. H. \& Fixman, M. Dilute solutions of branched polymers Ann. N. Y. Acad. Sci. 57, 334-352 (1953).

17 Irurzun, I. M. Hydrodynamic properties of regular star-branched polymer in dilute solution J. Polym. Sci. B: Polym. Phys. 35, 563-567 (1997).

18 Zimm, B. H. \& Stockmayer, W. H. The dimensions of chain molecules containing branches and rings. J. Chem. Phys. 17, 1301-1314 (1949).

19 Riseman, J. \& Kirkwood, J. G. The intrinsic viscosity, translational and rotatory diffusion constants of rod-like macromolecules in solution J. Chem. Phys. 18, 512-516 (1950).

20 Tchen, C.-M. Motion of small particles in skew shape suspended in a viscous liquid $J$. Appl. Phys. 25, 463-473 (1954).

21 Zwanzig, R. Translational diffusion in polymer solutions J. Chem. Phys. 45, 1858-1859 (1966).

22 Paul, E. \& Mazo, R. M. Translational diffusion coefficient of a plane polygonal polymer J. Chem. Phys. 48, 2378-2378 (1968).

23 Paul, E. \& Mazo, R. M. Hydrodynamic properties of a plane-polygonal polymer. According to Kirkwood-Riseman theory J. Chem. Phys. 51, 1102-1107 (1969). 
24 Yamakawa, H. \& Yamaki, J. Translational diffusion coefficients of plane-polygonal polymers: Application of the modified Oseen tensor J. Chem. Phys. 57, 1542-1546 (1972).

25 Huber, K., Burchard, W. \& Fetters, L. J. Dynamic light scattering of regular star-branched molecules Macromolecules 17, 541-548 (1984).

26 Norisuye, T. \& Fujita, H. Excluded-volume effects in dilute polymer solutions. XIII. Effects of chain stiffness Polym. J. 14 143-147 (1982).

27 Huber, K., Bantle, S., Lutz, P. \& Burchard, W. Hydrodynamic and thermodynamic behavior of short-chain polystyrene in toluene and cyclohexane at $34.5^{\circ} \mathrm{C}$ Macromolecules 18, 1461-1457 (1985).

28 Yamakawa, H. Some remarks on the transport theory for wormlike cylinder models Macromolecules 16, 1928-1931 (1983). 


\section{ASYMPTOTIC FORM FOR $D$ OF THE REGULAR THREE-ARM STAR IN THE ROD LIMIT}

In this appendix, we derive the asymptotic solutions in the limit of $L / d_{\mathrm{b}} \rightarrow \infty$ (thin or long rod limit) for $D^{(\mathrm{K})}$ and $D^{(\mathrm{KR})}$ of the KP regular three-arm star in the rod limit.

\section{Kirkwood value}

The asymptotic form of $D^{(\mathrm{K})}$ for the regular three-arm star in the thin rod limit may be straightforwardly derived from Eq. (4) with Eqs. (5), (6), (7), and (15).

In the case of the regular three-arm star, the summation in Eq. (4) may be rewritten in the form,

$$
\begin{aligned}
\sum_{\substack{i=0 \\
i \neq j}}^{n} \sum_{j=0}^{n}\left\langle R_{i j}{ }^{-1}\right\rangle= & 2\left[\sum_{i=0}^{2} \sum_{j=1}^{m}\left\langle R_{0(i m+j)}{ }^{-1}\right\rangle+\sum_{i=0}^{2} \sum_{j=1}^{m-1} \sum_{k=j+1}^{m}\left\langle R_{(i m+j)(i m+k)}{ }^{-1}\right\rangle\right. \\
& \left.+\sum_{i=0}^{1} \sum_{j=i+1}^{2} \sum_{k=1}^{m} \sum_{l=1}^{m}\left\langle R_{(i m+k)(j m+l)}{ }^{-1}\right\rangle\right] .
\end{aligned}
$$

Recall that $L=(n+1) d_{\mathrm{b}}$ and $m=n / 3$. In the limit of $L / d_{\mathrm{b}} \rightarrow \infty$, i.e., $m \rightarrow \infty$, we may perform the first and second summations on the right-had side of Eq. (39) as follows:

$$
\begin{gathered}
\sum_{i=0}^{2} \sum_{j=1}^{m}\left\langle R_{0(i m+j)}{ }^{-1}\right\rangle=\frac{3}{d_{\mathrm{b}}}\left[\ln m+\gamma_{\mathrm{E}}+\mathcal{O}\left(m^{-1}\right)\right], \\
\sum_{i=0}^{2} \sum_{j=1}^{m-1} \sum_{k=j+1}^{m}\left\langle R_{(i m+j)(i m+k)}{ }^{-1}\right\rangle=\frac{3 m}{d_{\mathrm{b}}}\left[\ln m+\gamma_{\mathrm{E}}-1+\mathcal{O}\left(m^{-1}\right)\right],
\end{gathered}
$$

where $\gamma_{\mathrm{E}}(=0.5772 \cdots)$ is the Euler constant. In this limit, the third summation on the right-had side of Eq. (39) may be converted to an integral and it may be calculated to be

$$
\sum_{i=0}^{1} \sum_{j=i+1}^{2} \sum_{k=1}^{m} \sum_{l=1}^{m}\left\langle R_{(i m+k)(j m+l)}{ }^{-1}\right\rangle=\frac{3 m}{d_{\mathrm{b}}}[\ln (3+2 \sqrt{3})-3(\ln 3) / 2-\ln (2-\sqrt{3})] .
$$

Then we have

$$
\lim _{\substack{L \rightarrow 0 \\ L / d_{\mathrm{b}} \rightarrow \infty}} \sum_{\substack{i=0 \\ i \neq j}}^{n} \sum_{j=0}^{n}\left\langle R_{i j}^{-1}\right\rangle=\frac{2 L}{d_{\mathrm{b}}{ }^{2}}\left[\ln \left(L / d_{\mathrm{b}}\right)+\mathcal{O}\left(\left[\ln \left(L / d_{\mathrm{b}}\right)\right]^{0}\right)\right]
$$

From Eq. (4) with Eq. (42), we obtain Eq. (26). 


\section{KR value}

In the thin rod limit, we may convert the summations in Eqs. (16) and (17) to integrals. In the case of the regular three-arm star, Eq. (16) with Eq. (17) may then be rewritten in the form,

$$
D^{(\mathrm{KR})}=k_{\mathrm{B}} T\left[3 \pi \eta_{0} L \int_{0}^{1} \psi(x) d x\right]^{-1},
$$

where $\psi(x)$ is the solution of the integral equation,

$$
\psi(x)=1-\frac{1}{2}\left[\int_{0}^{1} K_{0}(x, t) \psi(t) d t+2 \int_{0}^{1} K_{1}(x, t) \psi(t) d t\right] .
$$

In Eq. (44), $K_{0}(x, t)$ and $K_{1}(x, t)$ are the continuous versions of the mean reciprocal of the distance between the centers of two beads on the same arm and on the different arm, respectively, and they are explicitly given by

$$
\begin{array}{cc}
K_{0}(x, t)=|x-t|^{-1} & \text { for }|x-t| \geq 3 d_{\mathrm{b}} / L \\
=0 & \text { for }|x-t|<3 d_{\mathrm{b}} / L, \\
K_{1}(x, t)=\left(x^{2}+t^{2}+x t\right)^{-1 / 2} .
\end{array}
$$

From Eq. (44), the function $F(x)$ may be defined by

$$
\begin{aligned}
F(x) & =1-2 \phi(x) \\
& =\int_{0}^{1} K_{0}(x, t) \phi(t) d t+2 \int_{0}^{1} K_{1}(x, t) \phi(t) d t,
\end{aligned}
$$

where $\phi(x)=\psi(x) / 2$. We then expand $\phi(x)$ and $K_{k}(x, t)(k=1,2)$ in terms of the shifted Legendre polynomial $\tilde{P}_{l}(x)$ as follows,

$$
\begin{gathered}
\phi(x)=\sum_{i=0}^{\infty} \phi_{i} \tilde{P}_{i}(x), \\
K_{k}(x, t)=\sum_{i=0}^{\infty} \sum_{j=0}^{\infty} K_{k, i j} \tilde{P}_{i}(x) \tilde{P}_{j}(t), \quad(k=0,1)
\end{gathered}
$$

where $\tilde{P}_{l}(x)$ is defined by

$$
\tilde{P}_{l}(x)=(-1)^{l} P_{l}(2 x-1)
$$


with $P_{l}(x)$ the Legendre polynomial. We note that $\tilde{P}_{l}(x)$ satisfies the following orthogonality relation,

$$
\int_{0}^{1} \tilde{P}_{l}(x) \tilde{P}_{l^{\prime}}(x) d x=(2 l+1)^{-1} \delta_{l l^{\prime}}
$$

where $\delta_{l l^{\prime}}$ is the Kronecker delta. In Eqs. (48) and (49), the expansion coefficients $\phi_{i}$ and $K_{k, i j}$ may be given by

$$
\phi_{i}=(2 i+1) \int_{0}^{1} \phi(x) \tilde{P}_{i}(x) d x
$$

and

$$
K_{k, i j}=(2 i+1)(2 j+1) \int_{0}^{1} \int_{0}^{1} K_{k}(x, t) \tilde{P}_{i}(x) \tilde{P}_{j}(t) d x d t \quad(k=0,1),
$$

respectively. Substituting Eqs. (52) and (53) into the second line of Eq. (47) and carrying out the integrations, $F(x)$ may be rewritten in the form,

$$
F(x)=\sum_{i=0}^{\infty} \sum_{j=0}^{\infty}(2 j+1)^{-1}\left(K_{0, i j}+2 K_{1, i j}\right) \phi_{j} \tilde{P}_{i}(x) .
$$

It can be shown in the limit of $L / d_{\mathrm{b}} \rightarrow \infty$ that

$$
K_{0, i j}=(-1)^{i+j}(4 j+2) \delta_{i j} \ln \left(L / d_{\mathrm{b}}\right)+\mathcal{O}\left(\left[\ln \left(L / d_{\mathrm{b}}\right)\right]^{0}\right)
$$

and $K_{1, i j}=\mathcal{O}\left(\left[\ln \left(L / d_{\mathrm{b}}\right)\right]^{0}\right)$. Then we have

$$
F(x)=\left[2 \ln \left(L / d_{\mathrm{b}}\right)+\mathcal{O}\left(\left[\ln \left(L / d_{\mathrm{b}}\right)\right]^{0}\right)\right] \phi(x) .
$$

From the first line of Eq. (47) and Eq. (56) along with the relation $\phi(x)=\psi(x) / 2, \psi(x)$ may be written in the from,

$$
\psi(x)=\left[\ln \left(L / d_{\mathrm{b}}\right)+\mathcal{O}\left(\left[\ln \left(L / d_{\mathrm{b}}\right)\right]^{0}\right)\right]^{-1}
$$

Substituting of Eq. (57) into Eq. (43) and carrying out the integration over $x$, we obtain Eq. (30). 
Figure Legends

Figure 1 Plots of $g_{\mathrm{H}}^{(\mathrm{K})}\left(L, d_{\mathrm{b}}\right)$ and $g_{\mathrm{H}}^{(\mathrm{KR})}\left(L, d_{\mathrm{b}}\right)$ against $\log L$. The open and closed circles represent $g_{\mathrm{H}}^{(\mathrm{K})}$ and $g_{\mathrm{H}}^{(\mathrm{KR})}$, respectively, for $d_{\mathrm{b}}=0.001$ (pip up), 0.003 (pip rightup), 0.01 (pip right), 0.03 (pip right-down), 0.1 (pip down), 0.2 (pip left-down), 0.3 (pip left), and 0.4 (pip left-up). The dashed curves connect smoothly the theoretical values at constant $d_{\mathrm{b}}$. The lower and upper horizontal lines represent the random-coil limiting values $\sqrt{3} /(2 \sqrt{2}-1)(=0.947)$ of $g_{\mathrm{H}}^{(\mathrm{K})}$ and $0.96_{4}$ of $g_{\mathrm{H}}^{(\mathrm{KR})}$, respectively. The solid curves represent the values calculated from the interpolation formula for $g_{\mathrm{H}}^{(\mathrm{K})}$ (see the text).

Figure 2 Plots of $g_{\mathrm{H}}^{(\mathrm{KR})}$ against $L^{-1 / 2}$. All the symbols have the same meaning as those in Fig. 1. The dashed curves connect smoothly the theoretical values at constant $d_{\mathrm{b}}$ and the solid lines indicate the respective initial tangents.

Figure 3 Plots of $g_{\mathrm{H}, 0}^{(\mathrm{K})}\left(L / d_{\mathrm{b}}\right)$ against $\left[\ln \left(L / d_{\mathrm{b}}\right)\right]^{-1}$. The open circles represent the theoretical values. The horizontal line segment represent the asymptotic value 1 in the limit of $\left[\ln \left(L / d_{\mathrm{b}}\right)\right]^{-1} \rightarrow 0\left(L / d_{\mathrm{b}} \rightarrow \infty\right)$. The curve represents the values of the interpolation formula, the solid part indicating the range of $L / d_{\mathrm{b}} \gtrsim 10$ (see the text).

Figure 4 Plots of $g_{\mathrm{H}}^{(\mathrm{K})}\left(L, d_{\mathrm{b}}\right) / g_{\mathrm{H}, 0}^{(\mathrm{K})}\left(L / d_{\mathrm{b}}\right)$ against $\log L$. All the symbols have the same meaning as those in Fig. 1. The solid curves represent the values of the interpolation formula with the corresponding values of $d_{\mathrm{b}}$ (see the text).

Figure 5 Plots of $g_{\mathrm{H}}$ against the logarithm of $M_{\mathrm{w}}$ for regular three-arm star polystyrenes in cyclohexane at $34.5^{\circ} \mathrm{C}(\Theta)$. The open circles represent the experimental data obtained by Huber et.al. ${ }^{25}$ The curve represents the corresponding KP theory values, the solid part indicating the range of $L / d_{\mathrm{b}} \gtrsim 10$ (see text). 


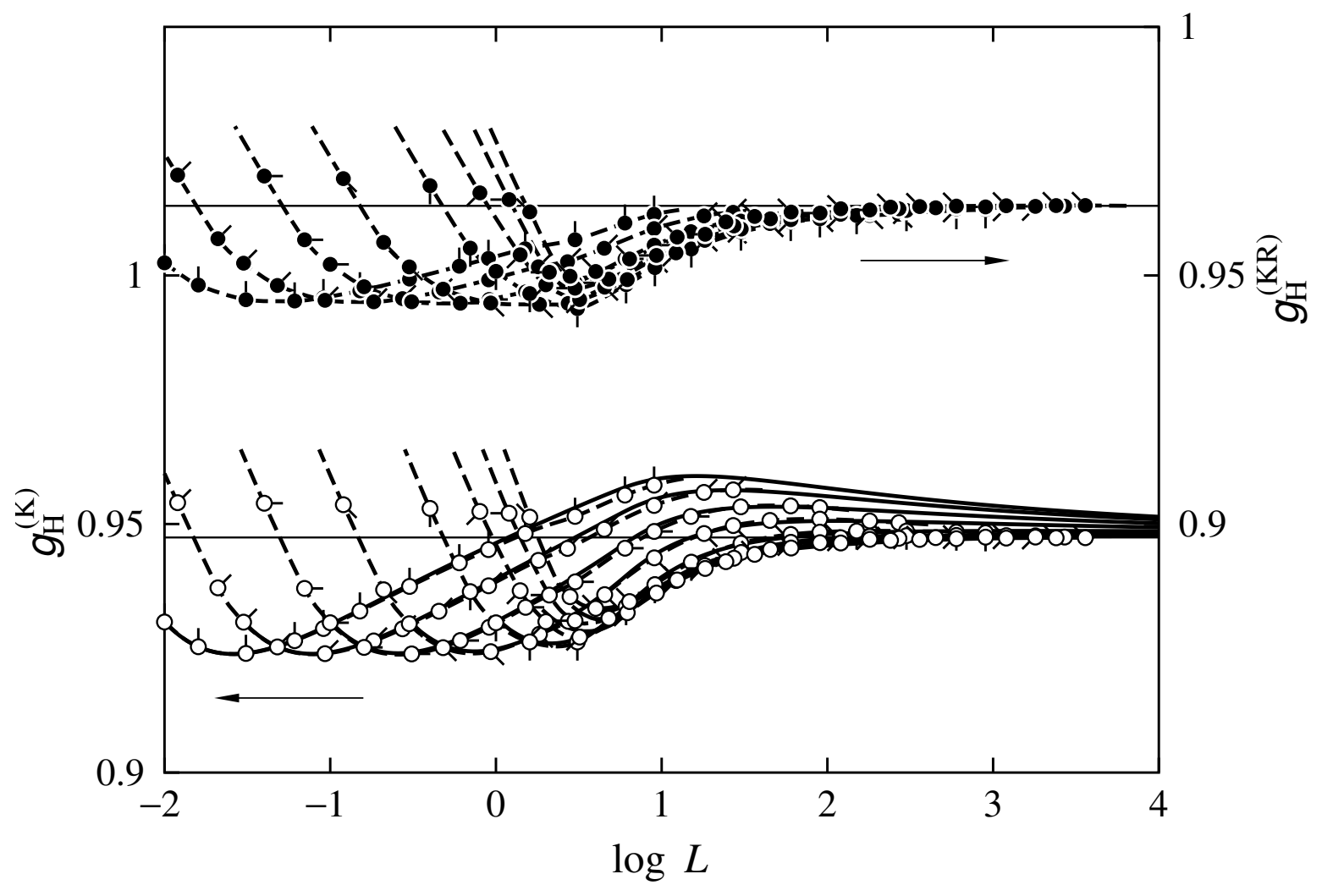

D. Ida, Figure 1 


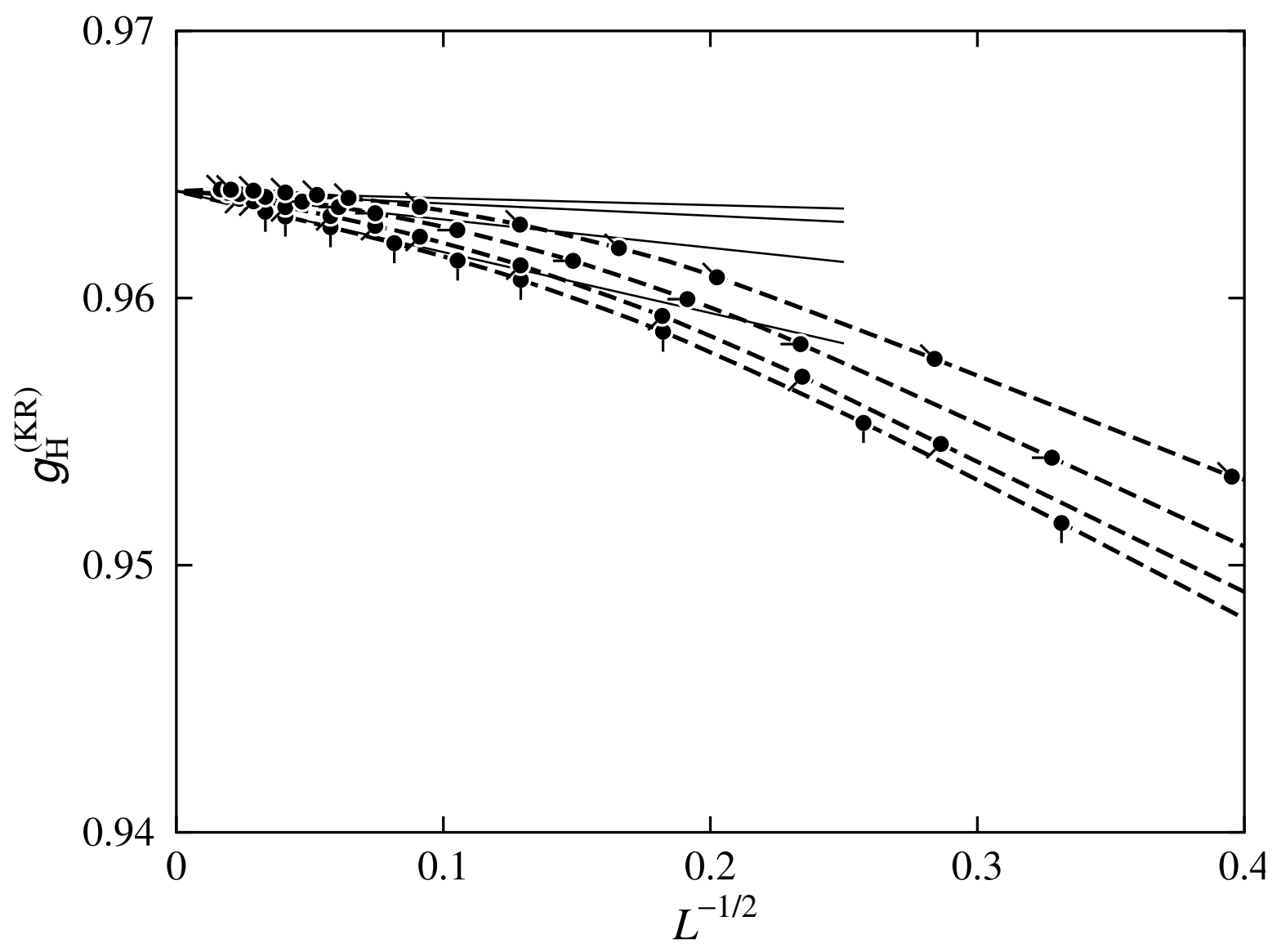

D. Ida, Figure 2 


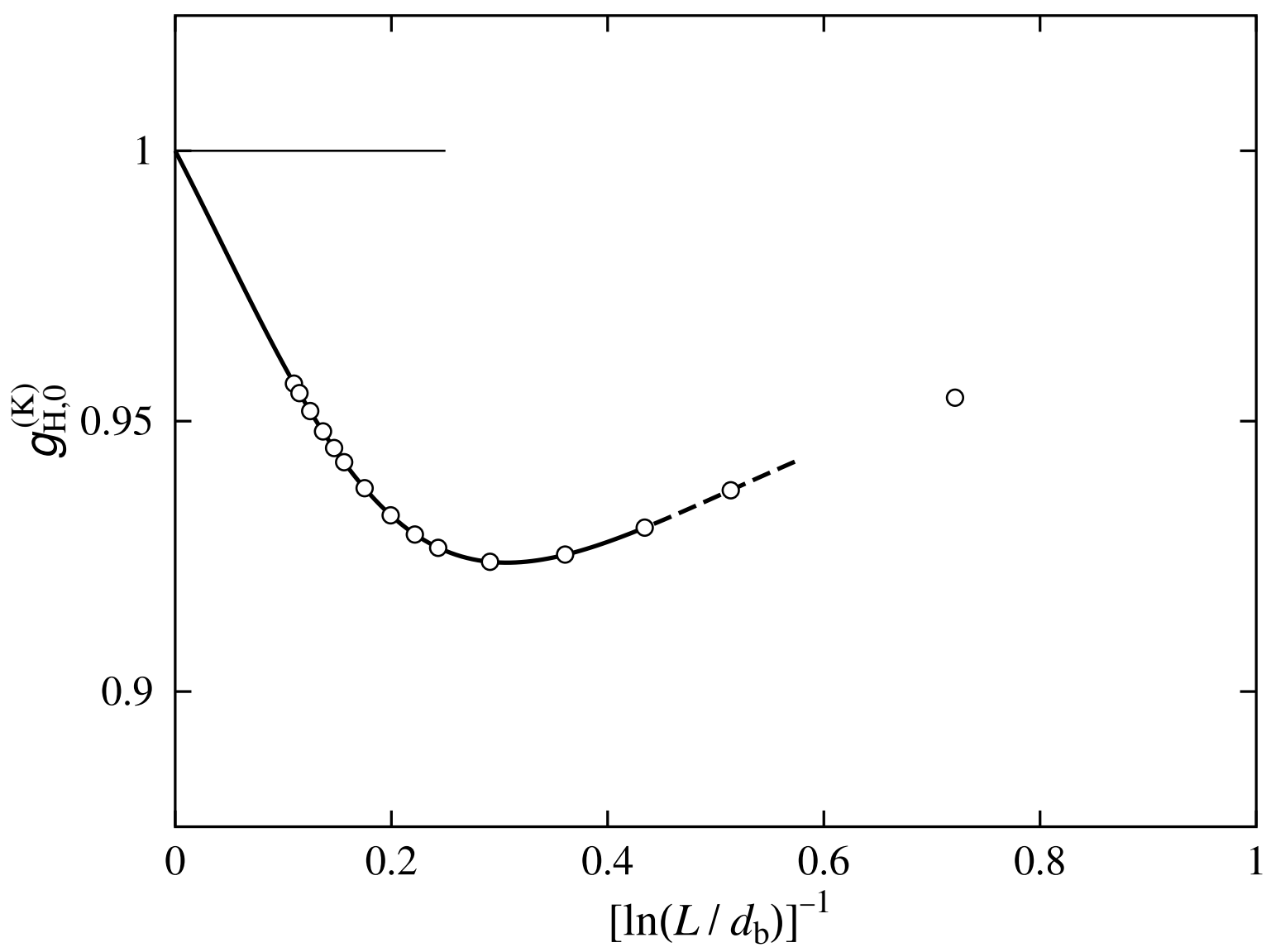

D. Ida, Figure 3 


\section{$-25-$}

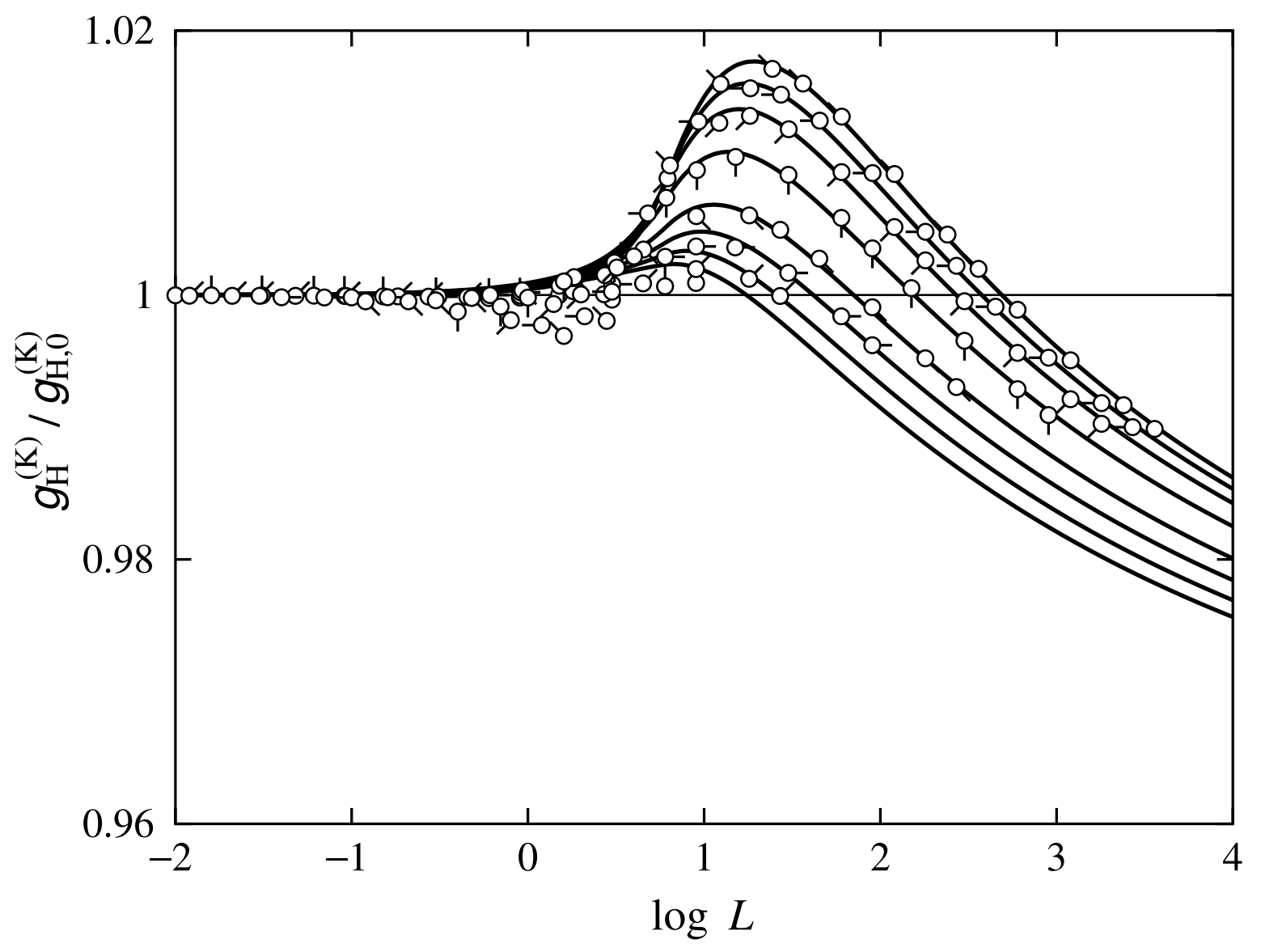

D. Ida, Figure 4 


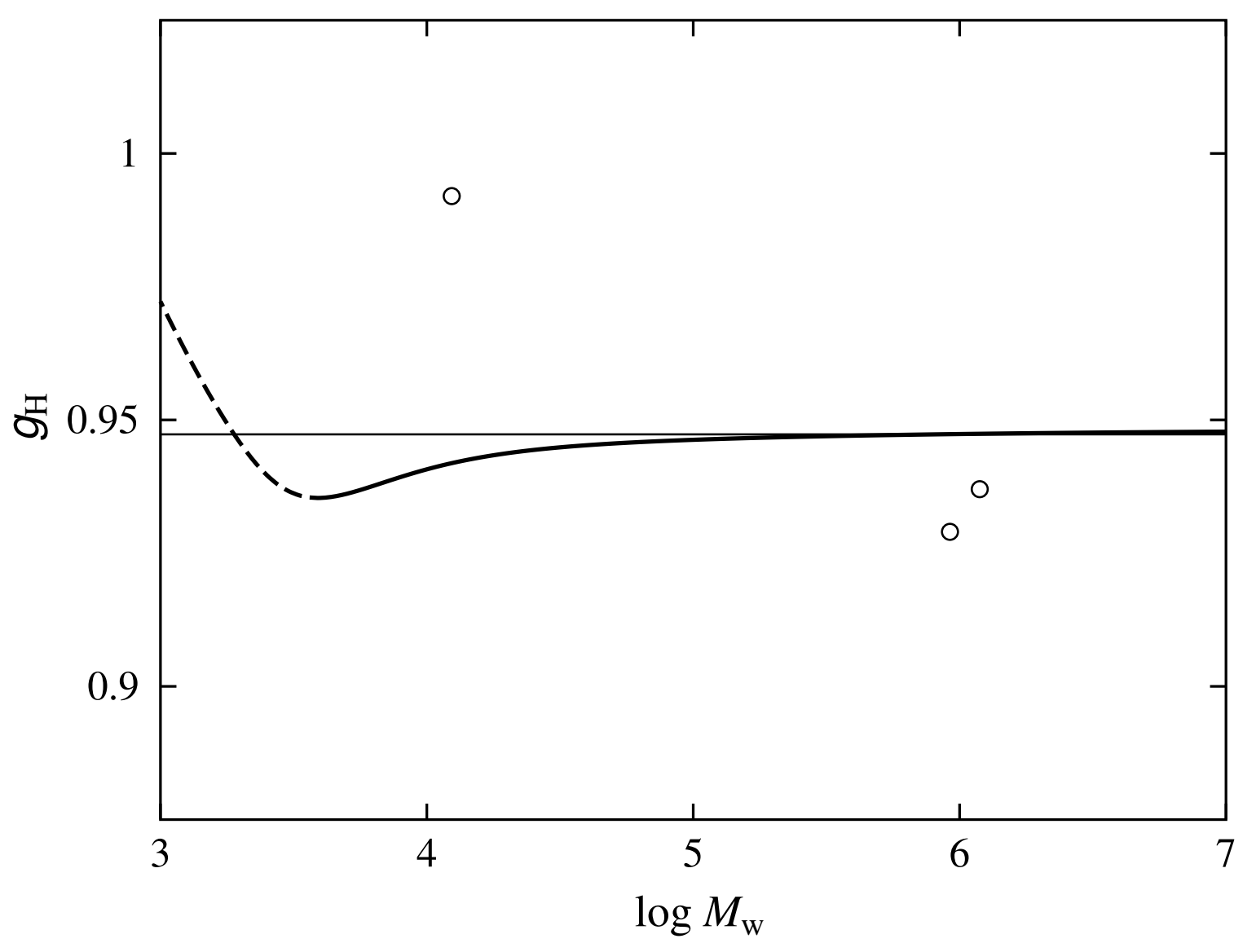

D. Ida, Figure 5 


\section{Graphical Abstract:}

The translational diffusion coefficient $D$ or (effective) hydrodynamic radius $R_{\mathrm{H}}$ of the regular three-arm star Kratky-Porod (KP) wormlike chain is evaluated. The behavior of the ratio $g_{\mathrm{H}}$ of $R_{\mathrm{H}}$ of the star and that of the corresponding linear chain is examined as a function of the (reduced) contour length $L$ and (reduced) hydrodynamic diameter $d_{\mathrm{b}}$.

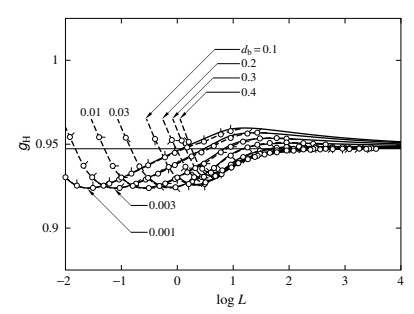

